\title{
Machine learning models to identify low adherence to influenza vaccination among Korean adults with cardiovascular disease
}

\author{
Moojung Kim ${ }^{1 \dagger}$, Young Jae Kim ${ }^{2 \dagger}$, Sung Jin Park ${ }^{2}$, Kwang Gi Kim ${ }^{2 *}$, Pyung Chun Oh ${ }^{3}$, Young Saing Kim ${ }^{3}$ \\ and Eun Young $\mathrm{Kim}^{4 *}$ (1)
}

\begin{abstract}
Background: Annual influenza vaccination is an important public health measure to prevent influenza infections and is strongly recommended for cardiovascular disease (CVD) patients, especially in the current coronavirus disease 2019 (COVID-19) pandemic. The aim of this study is to develop a machine learning model to identify Korean adult CVD patients with low adherence to influenza vaccination

Methods: Adults with CVD $(n=815)$ from a nationally representative dataset of the Fifth Korea National Health and Nutrition Examination Survey (KNHANES V) were analyzed. Among these adults, 500 (61.4\%) had answered "yes" to whether they had received seasonal influenza vaccinations in the past 12 months. The classification process was performed using the logistic regression (LR), random forest (RF), support vector machine (SVM), and extreme gradient boosting (XGB) machine learning techniques. Because the Ministry of Health and Welfare in Korea offers free influenza immunization for the elderly, separate models were developed for the $<65$ and $\geq 65$ age groups.

Results: The accuracy of machine learning models using 16 variables as predictors of low influenza vaccination adherence was compared; for the $\geq 65$ age group, XGB (84.7\%) and RF (84.7\%) have the best accuracies, followed by LR (82.7\%) and SVM (77.6\%). For the < 65 age group, SVM has the best accuracy (68.4\%), followed by RF (64.9\%), LR (63.2\%), and XGB (61.4\%).
\end{abstract}

Conclusions: The machine leaning models show comparable performance in classifying adult CVD patients with low adherence to influenza vaccination.

Keywords: Machine learning, Influenza vaccination, Cardiovascular disease

*Correspondence: kimkg@gachon.ac.kr; oneshot0229@gmail.com ${ }^{\dagger}$ Moojung Kim and Young Jae Kim contributed equally to this work ${ }^{\dagger}$ Kwang Gi Kim and Eun Young Kim contributed equally to this work

${ }^{2}$ Department of Biomedical Engineering, Gachon University College of Medicine, Incheon, South Korea

${ }^{4}$ Department of Radiology, Gil Medical Center, Gachon University College of Medicine, Incheon, South Korea

Full list of author information is available at the end of the article

\section{Background}

Influenza is an infectious disease of the respiratory system and the outbreak of influenza occurs worldwide in a seasonal manner, usually during the winter season in temperate climates. It is highly contagious and affects people via droplet contact. Influenza increases the morbidity and mortality among sufferers of cardiovascular disease (CVD), diabetes mellitus, asthma, obstructive pulmonary disease, and malignancy, who have higher 
chances of suffering from serious medical complications [1-3]. CVD is the leading global cause of death; approximately 17.9 million people died from CVD in 2016, accounting for $31 \%$ of all global deaths. Heart attack and stroke together contributed $85 \%$ of these deaths [4]. It is very important to recognize that CVD patients suffer from higher morbidity and mortality rates when they are infected with influenza. Several meta-analyses and systematic reviews have revealed a strong association between influenza infection and acute myocardial infarction $[3,5]$. These reviews strongly recommend influenza vaccination for CVD patients.

The coronavirus disease 2019 (COVID-19) is caused by severe acute respiratory syndrome coronavirus 2 (SARS$\mathrm{CoV}-2$ ) and was declared a pandemic by the World Health Organization (WHO), on March 11, 2020 [6]. In the COVID-19 pandemic era, patients with comorbidities have an increased case fatality ratio, including 6.0\% for hypertension, $7.3 \%$ for diabetes, and $10.5 \%$ for CVD [7]. With currently no proven vaccines or antiviral treatments, standard public health preventive efforts are being applied. To prevent the twindemic, where the COVID-19 pandemic and the 2020-21 influenza epidemic overlap, it is important to enhance influenza vaccination during the upcoming winter seasons.

In our previous study [8], we found that the vaccination coverage rate is low in non-elderly ( $<65$ years) CVD patients. To promote influenza vaccination coverage in CVD patients, it is necessary to identify a high risk population with low influenza vaccination adherence.

Machine learning algorithms build a model based on sample data, known as "training data", to make predictions or decisions without being explicitly programmed. As a subcategory of artificial intelligence, machine learning analyzes the patterns of the data and performs simultaneous tests on numerous variables to develop prediction models [9]. Therefore, this technique has a relative advantage over traditional statistical methods that can only address a small number of variables and cannot identify complex interactions between these variables.

The objective of this study was to create a model to predict influenza vaccination adherence using four different machine learning techniques: logistic regression (LR), support vector machine (SVM), random forest (RF), and extreme gradient boosting (XGB).

\section{Materials and methods}

\section{Data collection and preparation}

Data were obtained from the Fifth Korea National Health and Nutrition Examination Survey (KNHANES) conducted by the Korea Center for Disease Control and Prevention (KCDC) from January 2010 to December 2012. The dataset and questionnaire is provided with guidelines for calculating a health-related index through the KCDC online site (https://knhanes.cdc.go.kr/knhanes/eng/index .do).

KNHANES is a data resource operated by the KCDC since 1998. Researchers can access and use the data to support health-related studies. This nationally representative, cross-sectional survey includes approximately 10,000 participants every year and collects comprehensive data on their socioeconomic and nutritional status, lifestyle, physical activity, and health resource utilization through the three methods of health interviews, health examinations, and nutrition surveys. These three methods were conducted by professional staff members, including physicians and health interviewers. In the KNHANES, written informed consent was provided by every participant. The KNHANES survey was approved by the Institutional Review Board of the Korea Centers for Disease Control and Prevention (IRB No. 2010-02CON-21-C, 2011-02CON-06-C, $2012-$ 01EXP-01-2C). Further ethical approval for the use of KNHANES data are not required because publicly available datasets were used in this study.

From KNHANES V, 19,599 adults ( $>19$ years old) were selected by proportional allocation-systemic sampling with multistage stratification. Of the 19,599 participants in this sample, 17,872 participants have accessible information about their CVD and influenza vaccination status. Of these 17,872 participants, 815 had CVD; thus, 815 participants were included in the analysis (Table 1).

\section{CVD}

Participants were considered to have CVD if they have been diagnosed by a physician based on the survey question of "if they had been ever diagnosed with CVD (any of the following conditions: stroke, myocardial infarction, and angina pectoralis) by a doctor".

\section{Influenza vaccination}

As the dependent variable, the influenza vaccination status was assessed using the question "have you received an influenza vaccination within the past 1 year?", to which the participant answered "yes" or "No".

\section{Independent variables}

The independent variables included sociodemographic variables (sex, age, marital status, household income, education level, health insurance status, and private insurance), health-related lifestyle factors (height, weight, BMI, current smoking, drinking, exercise, and recent health screening), and health status (past history of hypertension, diabetes mellitus, hyperlipidemia, and cancer, and obesity and perceived health status). 
Table 1 Baseline characteristics of Korean adults with cardiovascular disease (CVD)

\begin{tabular}{|c|c|}
\hline Parameters & $\begin{array}{l}\text { Korean adults with } \\
\text { CVD }(\%)(n=815)\end{array}$ \\
\hline Sex, male & $53.0(2.2)$ \\
\hline Age (mean, yr) & $64.8(0.5)$ \\
\hline \multicolumn{2}{|l|}{ Age group } \\
\hline $19-49$ & $7.6(1.3)$ \\
\hline $50-64$ & $39.3(2.3)$ \\
\hline$\geq 65$ & $53.1(2.3)$ \\
\hline Spouse, yes & $77.5(1.8)$ \\
\hline \multicolumn{2}{|l|}{ Household income } \\
\hline Lowest quartile & $40.1(2.2)$ \\
\hline 2nd and 3rd quartile & $44.3(2.2)$ \\
\hline Highest quartile & $15.6(1.7)$ \\
\hline \multicolumn{2}{|l|}{ Education } \\
\hline Less than elementary school & $49.9(2.1)$ \\
\hline Middle or high school & $39.3(2.0)$ \\
\hline College and above & $10.8(1.3)$ \\
\hline \multicolumn{2}{|l|}{ Insurance } \\
\hline National health insurance & $93.6(1.1)$ \\
\hline Medical aid & $6.4(1.1)$ \\
\hline Private Insurance, yes & $40.3(2.0)$ \\
\hline Body mass index (mean, kg/m²) & $24.5(0.1)$ \\
\hline Obesity (BMI $\left.\geq 25 \mathrm{~kg} / \mathrm{m}^{2}\right)$ & $41.6(2.2)$ \\
\hline Alcohol drinker & $14.8(1.9)$ \\
\hline Current smoker & $18.9(1.9)$ \\
\hline Routine exerciser & $43.1(2.2)$ \\
\hline Hypertension & $62.2(2.0)$ \\
\hline Diabetes mellitus & $25.8(1.8)$ \\
\hline Hyperlipidemia & $32.7(2.1)$ \\
\hline Cancer & $5.0(0.8)$ \\
\hline \multicolumn{2}{|l|}{ Perceived health status } \\
\hline Good to very good & $13.4(1.4)$ \\
\hline Normal & $37.9(2.1)$ \\
\hline Poor to very poor & $48.7(2.1)$ \\
\hline Health screening & $61.0(2.1)$ \\
\hline Influenza vaccination & $61.4(2.2)$ \\
\hline
\end{tabular}

Abbreviations: BMI, body mass index

The socio-demographic variables comprised the current age (19-49, 50-64, and $\geq 65$ years), marital status (unmarried, separated, widowed, and divorced subjects were allocated a "no spouse" status), national insurance state (national health insurance, medical aid, or neither), and private health insurance state (yes, no). The household income level was classified into national quartile groups (lowest, 2nd, 3rd and highest quartile groups). The education background was divided into
Table 2 Prediction model development after dividing dataset into two age groups

\begin{tabular}{lllll}
\hline $\begin{array}{l}\text { Korean adults with cardiovascular } \\
\text { disease }\end{array}$ & Training data & Test data & Total \\
\hline$\geq 65$ years & Influenza vaccination, no & 70 & 8 & 78 \\
& $\begin{array}{l}\text { Influenza vaccination, } \\
\text { yes }\end{array}$ & 376 & 42 & 418 \\
& Total & 446 & 50 & 496 \\
$<65$ years & Influenza vaccination, no & 157 & 17 & 174 \\
& Influenza vaccination, & 97 & 11 & 108 \\
& yes & & & \\
& Total & 254 & 28 & 282 \\
\hline
\end{tabular}

three categories, namely, less than elementary school, middle/high school, and college or above.

The health-related lifestyle factors included height, weight, and BMI, all of which are continuous variables. The BMI was calculated by dividing the weight $(\mathrm{kg})$ by the square of the height $\left(\mathrm{m}^{2}\right)$. The currently smoking variable has two categorical values, namely, yes and no. The recent health screening variable was set to "yes" for participants who replied that they had undergone health screening within the last two years. The exercise variable was set to "yes" for participants who walked or ran for at least 30 min more than 3 times a week.

The health status encompassed participants' past histories of hypertension, diabetes mellitus, hyperlipidemia, and cancer as categorical "yes" or "no" variables. Obesity was also a categorical variable and was divided into two groups: BMI above $25 \mathrm{~kg} / \mathrm{m}^{2}$ and BMI below $25 \mathrm{~kg} / \mathrm{m}^{2}$. Finally, the perceived health status was divided into three categories: good, normal, and bad.

\section{Models to predict influenza vaccination status}

A prediction model for the influenza vaccination status was developed separately for two age groups: older than or equal to 65 years old, and younger than 65 years old. The alcohol consumption categorical variable was excluded from the model because there were missing data for this variable. Therefore, out of the 815 people with CVD (500 people with vaccination and 315 people without vaccination), 778 were ultimately selected, as shown in Table 2 and Fig. 1.

To prevent overfitting, a tenfold cross validation was used. The original sample dataset was randomly partitioned into 10 equal sized subsamples. Out of the 10 groups, 9 groups were used as the training sets for prediction models. The remaining group was used as the test dataset. Finally, Four models were developed with the training datasets using the LR, SVM, RF, and XGB machine learning techniques. 


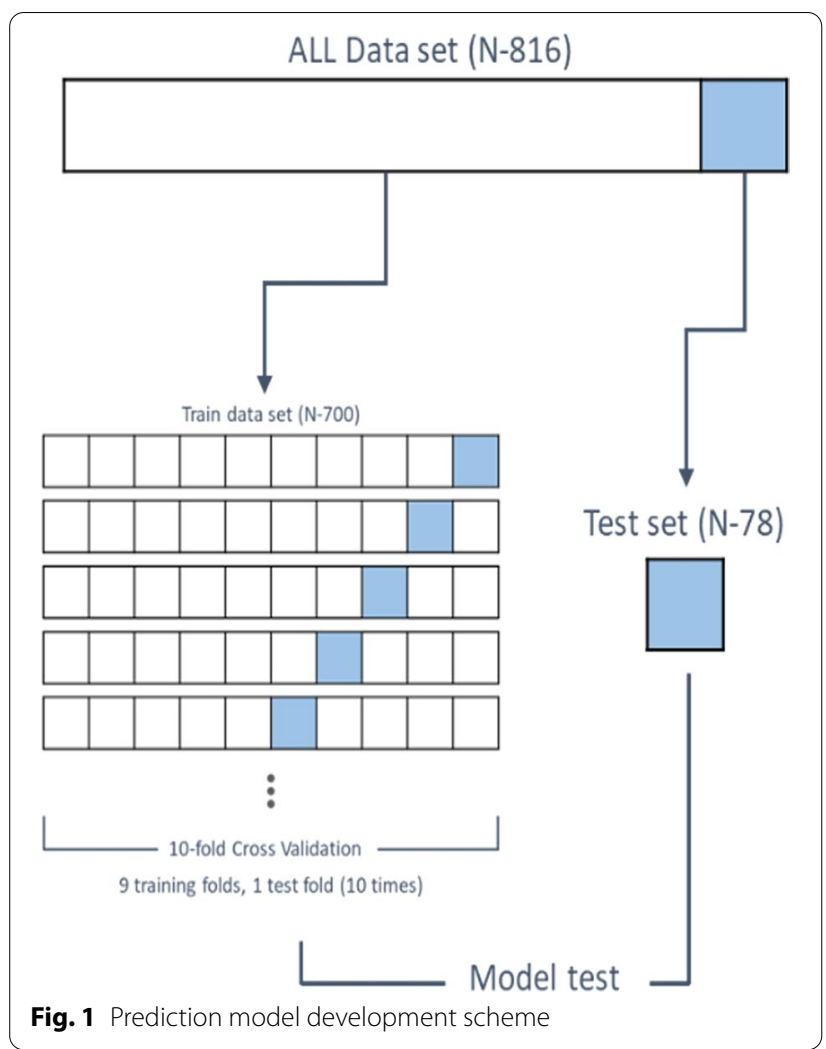

\section{Statistical analysis}

The results are presented as percentages for the categorical variables and as means ( \pm standard deviation) for the continuous variables. Logistic regression analysis was used to identify the factors associated with influenza vaccination adherence in the two age groups of above and below 65 years of age.

The machine learning models for classifying adherence to influenza vaccination were also developed separately for the two different age groups. A confusion matrix was used for measuring the diagnostic performance of each models in terms of the true positive (TP), false negative (FN), false positive (FP), and true negative (TN) rates, and the accuracy in predicting vaccination adherence among CVD patients. In addition, the area under the receiver operating characteristic curve (AUC) for each machine learning technique model was calculated to evaluate the general prediction performance on the test dataset.

The development of machine learning models and the analysis of the diagnostic performance were implemented using the open-source statistical software Python version 3.6.0. P-values of less than 0.05 (twosided) were considered to be significant.

\section{Results}

The univariable and multivariable logistic regression analysis results are summarized in Table 3 and Table 4. Among CVD patients aged $\geq 65$ years, there are two significant variables associated with influenza vaccination, namely, the sex and national insurance state. Males (odds ratio [OR], 0.34 within a $95 \%$ confidence interval [95\% CI], 0.14-0.84) and recipients of medical aid (OR, 0.34; 95\% CI, 0.15-0.79) were less likely to receive influenza vaccination according to Table 3. In addition, recent health screening (OR, 1.97; 95\% CI, 1.15-3.35) is associated with high adherence to influenza vaccination.

In CVD patients aged < 65 years, age (OR, 1.06; $95 \%$ $\mathrm{CI}, 1.01-1.12)$ and recent health screening (OR, 3.01; 95\% CI, 1.56-5.79) are the two most significant factors associated with influenza vaccination (Table 4). Older CVD patients who have undergone recent health screening in this group are more likely to show high adherence to influenza vaccination.

Table 5 and Fig. 2 show the performance of the LR and the machine learning models in predicting influenza vaccination adherence on the test datasets. The AUC of LR is comparable to that of the three machine learning models. RF shows the best performance, with an AUC of 0.643 for age $\geq 65$ years and AUC of 0.740 for age $<65$ years; however, the difference from the other models is not significant.

\section{Discussion}

Since patients with CVD present higher morbidity and mortality when infected by seasonal influenza, it is strongly recommended for CVD patients to receive influenza vaccination. However, the influenza vaccination adherence for this population is not high, especially among the non-elderly [8]. Recently, the use of machine learning has received increasing attention over time, especially in medical science where a tremendous amount of data has been generated from research and clinical practice $[10,11]$.

Using data from KHANES V, 815 adult CVD patients with accessible data were selected to develop machine learning model to identify low adherence to influenza vaccination. We developed several classification model using different machine learning techniques. With these model, CVD patients at risk of influenza infection can be identified so that health care and the promotion of influenza vaccination can be enhanced for these high-risk patients. Because a well-known factor associated with influenza vaccination is the age [8], since the vaccination is free for adults above the age of 65 in Korea, separate classification models were 
Table 3 Univariate and multivariate logistic regression analysis to identify factors associated with influenza vaccination status among Korean adults with cardiovascular disease ( $\geq 65$ years)

\begin{tabular}{|c|c|c|c|c|c|c|c|c|c|}
\hline & Crude OR & $(95 \% \mathrm{Cl})$ & Adjusted OR & $(95 \% \mathrm{Cl})$ & & Crude OR & $(95 \% \mathrm{Cl})$ & Adjusted OR & $(95 \% \mathrm{Cl})$ \\
\hline Sex & & & & & Sex & & & & \\
\hline Female & Reference & & Reference & & Female & Reference & & Reference & \\
\hline Male & 0.71 & $(0.43-1.16)$ & 0.34 & $(0.14-0.84)$ & Male & 0.47 & $(0.29-0.77)$ & 0.45 & $(0.18-1.09)$ \\
\hline Age & 1.01 & $(0.96-1.05)$ & 1.03 & $(0.98-1.09)$ & Age & 1.07 & $(1.03-1.12)$ & 1.06 & $(1.01-1.12)$ \\
\hline Height & 1 & $(0.97-1.03)$ & 1.14 & $(0.91-1.43)$ & Height & 0.97 & $(0.95-1.0)$ & 1 & $(0.76-1.31)$ \\
\hline Weight & 1 & $(0.98-1.03)$ & 0.87 & $(0.65-1.16)$ & Weight & 1 & $(0.98-1.03)$ & 1.03 & $(0.74-1.45)$ \\
\hline $\begin{array}{l}\text { Body mass index, } \\
\mathrm{kg} / \mathrm{m}^{2}\end{array}$ & 1.02 & $(0.94-1.1)$ & 1.44 & $(0.71-2.92)$ & $\begin{array}{l}\text { Body mass index, } \\
\mathrm{kg} / \mathrm{m}^{2}\end{array}$ & 1.08 & $(1.0-1.18)$ & 1.05 & $(0.42-2.59)$ \\
\hline Current smoking & & & & & Current smoking & & & & \\
\hline No & Reference & & Reference & & No & Reference & & Reference & \\
\hline Yes & 0.51 & $(0.26-0.99)$ & 0.62 & $(0.3-1.28)$ & Yes & 0.36 & $(0.19-0.69)$ & 0.51 & $(0.24-1.1)$ \\
\hline $\begin{array}{l}\text { Recent health } \\
\text { screen }\end{array}$ & & & & & $\begin{array}{l}\text { Recent health } \\
\text { screen }\end{array}$ & & & & \\
\hline No & Reference & & Reference & & No & Reference & & Reference & \\
\hline Yes & 2.13 & $(1.31-3.47)$ & 1.97 & $(1.15-3.35)$ & Yes & 3.37 & $(1.87-6.07)$ & 3.01 & $(1.56-5.79)$ \\
\hline Spous_yes & & & & & Spous_yes & & & & \\
\hline No & Reference & & Reference & & No & Reference & & Reference & \\
\hline Yes & 1.52 & $(0.92-2.53)$ & 1.94 & $(0.96-3.93)$ & Yes & 0.5 & $(0.23-1.08)$ & 0.57 & $(0.24-1.36)$ \\
\hline Insurance & & & & & Insurance & & & & \\
\hline $\begin{array}{l}\text { National health } \\
\text { insurance }\end{array}$ & Reference & & Reference & & $\begin{array}{l}\text { National health } \\
\text { insurance }\end{array}$ & Reference & & Reference & \\
\hline $\begin{array}{l}\text { Medical aid } \\
\text { Insurance_private }\end{array}$ & 0.32 & $(0.15-0.67)$ & 0.34 & $(0.15-0.79)$ & $\begin{array}{l}\text { Medical aid } \\
\text { Insurance_private }\end{array}$ & 0.8 & $(0.2-3.27)$ & 1.01 & $(0.19-5.47)$ \\
\hline No & Reference & & Reference & & No & Reference & & Reference & \\
\hline Yes & 1.12 & $(0.62-2.04)$ & 1.16 & $(0.61-2.2)$ & Yes & 1.31 & $(0.78-2.2)$ & 1.53 & $(0.85-2.76)$ \\
\hline Obesity & & & & & Obesity & & & & \\
\hline $\begin{array}{l}\text { Under BMI } 25 \mathrm{~kg} / \\
\mathrm{m}^{2}\end{array}$ & Reference & & Reference & & $\begin{array}{l}\text { under BMI } 25 \mathrm{~kg} / \\
\mathrm{m}^{2}\end{array}$ & Reference & & Reference & \\
\hline$\geq \mathrm{BMI} 25 \mathrm{~kg} / \mathrm{m}^{2}$ & 1.07 & $(0.65-1.75)$ & 1.07 & $(0.47-2.47)$ & $\geq \mathrm{BMI} 25 \mathrm{~kg} / \mathrm{m}^{2}$ & 1.25 & $(0.77-2.03)$ & 0.74 & $(0.31-1.74)$ \\
\hline Routine exerciser & & & & & Routine exerciser & & & & \\
\hline No & Reference & & Reference & & No & Reference & & Reference & \\
\hline Yes & 0.8 & $(0.49-1.3)$ & 0.85 & $(0.51-1.44)$ & Yes & 1.16 & $(0.71-1.89)$ & 1.14 & $(0.67-1.97)$ \\
\hline Hypertension & & & & & Hypertension & & & & \\
\hline No & Reference & & Reference & & No & Reference & & Reference & \\
\hline Yes & 0.94 & $(0.54-1.64)$ & 0.83 & $(0.46-1.51)$ & Yes & 1.57 & $(0.97-2.55)$ & 1.22 & $(0.69-2.15)$ \\
\hline Diabetes mellitus & & & & & Diabetes mellitus & & & & \\
\hline No & Reference & & Reference & & No & Reference & & Reference & \\
\hline Yes & 1.03 & $(0.61-1.75)$ & 1.23 & $(0.7-2.17)$ & Yes & 1.55 & $(0.87-2.76)$ & 1.59 & $(0.81-3.11)$ \\
\hline Hyperlipidemia & & & & & Hyperlipidemia & & & & \\
\hline No & Reference & & Reference & & No & Reference & & Reference & \\
\hline Yes & 1.2 & $(0.69-2.07)$ & 1.02 & $(0.56-1.84)$ & Yes & 1.34 & $(0.81-2.21)$ & 1.26 & $(0.7-2.27)$ \\
\hline History of cancer & & & & & History of cancer & & & & \\
\hline No & Reference & & Reference & & No & Reference & & Reference & \\
\hline Yes & 1.69 & $(0.58-4.9)$ & 2.14 & $(0.65-7.03)$ & Yes & 1.08 & $(0.3-3.91)$ & 1.12 & $(0.28-4.49)$ \\
\hline
\end{tabular}

Table 4 Multivariate logistic regression analysis to identify factors associated with influenza vaccination status among Korean adults with cardiovascular disease ( $<65$ years) 
Table 5 Confusion matrix for prediction models (Test dataset for the over-65 age group and under 65 age group)

\begin{tabular}{|c|c|c|c|c|c|c|}
\hline & \multicolumn{6}{|c|}{ Korean adult with cardiovascular disease ( $\geq 65$ yrs) } \\
\hline & $\mathrm{TP}$ & FN & $\mathrm{FP}$ & TN & Accuracy & AUC \\
\hline$L R$ & 1 & 14 & 3 & 80 & 0.827 & 0.639 \\
\hline SVM & 2 & 13 & 9 & 74 & 0.776 & 0.572 \\
\hline RF & 0 & 15 & 0 & 83 & 0.847 & 0.643 \\
\hline \multirow[t]{3}{*}{$X G B$} & 0 & 15 & 0 & 83 & 0.847 & 0.607 \\
\hline & \multicolumn{6}{|c|}{ Korean adult with cardiovascular disease ( $<65$ yrs) } \\
\hline & $\mathrm{TP}$ & FN & $\mathrm{FP}$ & TN & Accuracy & AUC \\
\hline$L R$ & 35 & 0 & 21 & 1 & 0.632 & 0.739 \\
\hline SVM & 31 & 4 & 14 & 8 & 0.684 & 0.697 \\
\hline $\mathrm{RF}$ & 31 & 4 & 16 & 6 & 0.649 & 0.74 \\
\hline$X G B$ & 35 & 0 & 22 & 0 & 0.614 & 0.67 \\
\hline
\end{tabular}
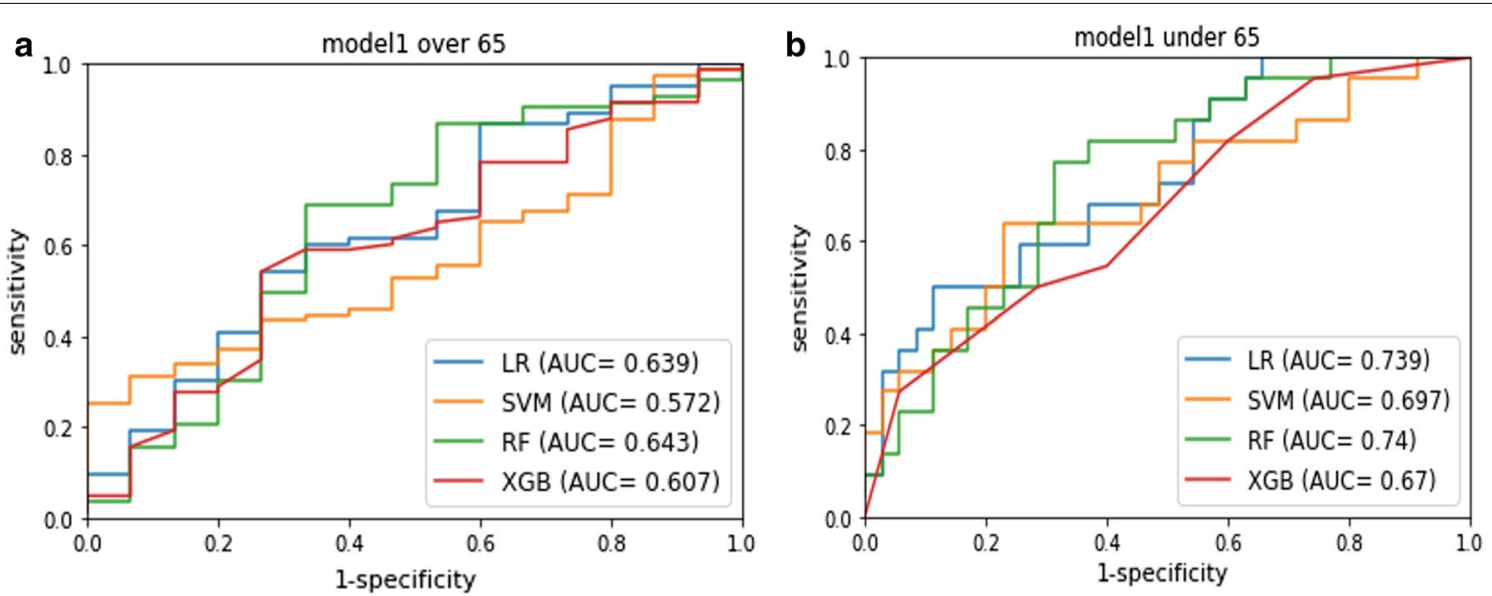

Fig. 2 Receiver operation characteristics. Abbreviations: LR, logistic regression; SVM, support vector machine; RF, Random Forest; XGB, extreme gradient boosting; TP, true positive; FN, false negative; FP, false positive; TN, true negative; AUC, area under ROC curve

Among the elderly CVD patients (age $\geq 65$ years), the sex and national insurance type significantly affect influenza vaccination adherence. On the other hand, for the $<65$ age group, the age and recent health screening status are significant factors. Using socio-demographic variables, health-related lifestyle factors, and health status, prediction models were generated using four machine learning techniques, namely, LR, SVM, $\mathrm{RF}$, and XGB. Using a tenfold cross validation, the dataset was split into ten equally sized random groups; nine groups were used as the training sets for the prediction models, and the remaining group was used as the test dataset, and to train the four prediction models. The diagnostic performance of LR is comparable to that of the three machine learning models, and the RF prediction model shows the best AUC for predicting the vaccination status among CVD patients in both age groups.

From KNHANES III to VI, influenza vaccination rates have steadily increased from 2005 to 2014 in South Korea and high vaccination coverage was associated with female gender, rural residence, low education level, high income, and increasing number of chronic diseases [12]. Factors associated with influenza vaccination coverage was also analyzed among elderly, patients with diabetes, chronic obstructive pulmonary disease, asthma, CVD, cancer survivors using the traditional multivariable logistic regression analysis on KNHANES dataset $[8,13-17]$. In the present study, we used machine learning techniques to identify factors associated with low adherence to influenza vaccination among Korean adults with CVD. Machine learning has 
advantage over traditional statistical methods since it can address a lot of variable information and can easily address complex interactions between these variables.

Influenza vaccination plays an important role in protecting high-risk population, which is a group that is particularly vulnerable for COVID-19. A number of papers that highlight the significant benefits of influenza vaccination in the current COVID-19 pandemic [18]. Influenza vaccination reduced COVID-19 infection risk in COVID-19 infection prediction model [19]. The influenza vaccination would enhance the management of respiratory outbreaks coinciding with the peak flu season, thus enabling more efficient use of healthcare resources [20].

\section{Limitation}

There are several limitations to this study. First, this study has a small sample size. A reliable way to validate the performance of a machine learning model is to train the model with available data and assess its classification performance using newly collected data or a separate dataset. Using unseen data to evaluate a machine learning model gives an unbiased estimate of its performance. We developed machine learning models to classify 16 survey-based variables (most of the variables were binary variables) in a total of 778 adults with CVD using data from KHANES. These 778 adults were subdivided into two groups according to their ages: adults aged $\geq 65$ years $(\mathrm{n}=496)$ and adults aged $<65$ years $(\mathrm{n}=282)$. Because of the small sample size, the receiver operating characteristics (ROC) curve is not a smooth curve but a step graph. When validation with a separate dataset is not feasible because of the small sample size, K-Fold cross-validation is very economical as it uses all the data for training and also reuses all the data for validation. Cross-validation is a common solution when the available datasets are limited; instead of training a fixed model only once as in the train/test split, several models are iteratively developed using different portions of the data on the cross-validation method. Second, the dataset was imbalanced. Imbalanced data is an unequally distributed dataset in which a certain class of data is significantly larger in quantity than the other data classes [21]. Owing to this disproportionate dataset, the prediction model tends to have good accuracy on the majority class data but poor accuracy on the rest of the data. That is, the prediction model can be inaccurate and biased towards the majority class data. In this study, among the 778 CVD patients, 526 received influenza vaccination and the remaining 252 did not. Because the proportion of people with influenza vaccination is quite different between the two age groups, there is a possibility of learning bias in the machine learning models. Due to these limitations, further studies using larger sample sizes and balanced datasets will be needed.

\section{Future directions}

Influenza vaccination is an important public health goal for protecting high-risk cardiovascular patients in the context of the ongoing COVID-19 pandemic. It is very important to identify the low adherence group and implement strategies to increase influenza vaccination coverage.

\section{Conclusion}

Machine learning models showed comparable performance in identifying low influenza vaccination adherence among CVD patients. Machine learning model might be used to enhance the health care for high-risk CVD patients by identifying patients at risk of influenza infection and promoting influenza vaccination to them.

\section{Abbreviations}

CVD: Cardiovascular disease; KNHANES: Korea National Health and Nutrition Examination Survey; LR: Logistic regression; RF: Random forest; SVM: Support vector machine; XGB: Extreme gradient boosting; WHO: World Health Organization; KCDC: Korea Center for Disease Control and Prevention; BMI: Body mass index; TR: True positive; FN: False negative; FP: False positive; TN: True negative; AUC: Receiver operating characteristic curve; OR: Odds ratio; Cl: Confidence interval; ROC: Receiver operating characteristics.

\section{Acknowledgements \\ Not applicable.}

\section{Authors' contributions}

EYK was agreed to be accountable for all aspects of the work in ensuring that questions related to the accuracy or integrity of any part of the work are appropriately investigated and resolved and drafted the manuscript. MK and EYK made substantial contributions to conception and design and revised the manuscript critically for important intellectual content; YJK, SJP, KGK, PCO made substantial contributions to acquisition of data; MK and YSK made substantial contributions to analysis and interpretation of data. MK and EYK given final approval of the version to be published. All authors read and approved the final manuscript.

\section{Funding}

Author who received: EYK. This research was supported by MD-PhD research grant through the Korea ResearchDriven Hospital (grant 2018-5287) and the National research foundation of Korea (grant NRF-2018R1C1B5086352). The funder had no role in study design, data collection and analysis, decision to publish, or preparation of the manuscript.

\section{Availability of data and materials}

The public access to the database is open; the dataset and questionnaire is provided with guidelines for calculating a health-related index through the KCDC online site (https://knhanes.cdc.go.kr/knhanes/eng/index.do).

\section{Ethics approval and consent to participate}

The KNHANES survey was approved by the Institutional Review Board of the Korea Centers for Disease Control and Prevention (IRB No. 2010-02CON-21-C, 2011-02CON-06-C, 2012-01EXP-01-2C). Further ethical approval for the use of KNHANES data are not required because publicly available datasets were used in this study. 


\section{Consent for publication \\ Not applicable.}

\section{Competing interests}

The author reports no conflicts of interest in this work.

\section{Author details}

${ }^{1}$ School of Medicine, Gachon University, Incheon, South Korea. ${ }^{2}$ Department of Biomedical Engineering, Gachon University College of Medicine, Incheon, South Korea. ${ }^{3}$ Department of Internal Medicine, Gil Medical Center, Gachon University College of Medicine, Incheon, South Korea. ${ }^{4}$ Department of Radiology, Gil Medical Center, Gachon University College of Medicine, Incheon, South Korea.

Received: 22 September 2020 Accepted: 18 February 2021

Published online: 09 March 2021

\section{References}

1. Grohskopf LA, Sokolow LZ, Broder KR, Walter EB, Bresee JS, Fry AM, Jernigan DB. Prevention and Control of Seasonal Influenza with Vaccines: Recommendations of the Advisory Committee on Immunization Practices - United States, 2017-18 Influenza Season. MMWR Recomm Rep. 2017;66(2):1-20

2. Simonsen L, Clarke MJ, Williamson GD, Stroup DF, Arden NH, Schonberger LB. The impact of influenza epidemics on mortality: introducing a severity index. Am J Public Health. 1997;87(12):1944-50.

3. Kwong JC, Schwartz KL, Campitelli MA, Chung H, Crowcroft NS, Karnauchow T, Katz K, Ko DT, McGeer AJ, McNally D. Acute myocardial infarction after laboratory-confirmed influenza infection. N Engl J Med. 2018;378(4):345-53

4. Mortality GBD. Causes of Death C: Global, regional, and national life expectancy, all-cause mortality, and cause-specific mortality for 249 causes of death, 1980-2015: a systematic analysis for the Global Burden of Disease Study 2015. Lancet. 2016;388(10053):1459-544.

5. Barnes M, Heywood AE, Mahimbo A, Rahman B, Newall AT, Macintyre CR. Acute myocardial infarction and influenza: a meta-analysis of casecontrol studies. Heart. 2015;101(21):1738-47.

6. Wang C, Horby PW, Hayden FG, Gao GF. A novel coronavirus outbreak of global health concern. Lancet. 2020;395(10223):470-3.

7. Bansal M. Cardiovascular disease and COVID-19. Diabetes Metab Syndr. 2020;14(3):247-50.

8. Kim EY, Ko JH, Kim YS, Oh PC. Prevalence and associated factors of influenza vaccination coverage in Korean adults with cardiovascular disease. Medicine (Baltimore). 2020:99(1):e18540.
9. Kotsiantis SB, Zaharakis I, Pintelas P. Supervised machine learning: a review of classification techniques. Emerg Artif Intell Appl Comput Eng. 2007:160:3-24

10. Obermeyer Z, Emanuel EJ. Predicting the future-big data, machine learning, and clinical medicine. N Engl J Med. 2016;375(13):1216.

11. Vayena E, Blasimme A, Cohen IG. Machine learning in medicine: addressing ethical challenges. PLoS Med. 2018:15:11.

12. Seo J, Lim J. Trends in influenza vaccination coverage rates in South Korea from 2005 to 2014: effect of public health policies on vaccination behavior. Vaccine. 2018;36(25):3666-73.

13. Shin $H Y$, Chung JH, Hwang HJ, Kim TH. Factors influencing on influenza vaccination and its trends of coverage in patients with diabetes in Korea: a population-based cross-sectional study. Vaccine. 2018;36(1):91-7.

14. Chung JH, Kim TH, Han CH. Factors influencing influenza vaccination among South Korean adult asthma patients: A nationwide populationbased cross-sectional study. J Asthma. 2018;55(3):294-9.

15. Shin $H Y$, Hwang $H J$, Chung JH. Factors influencing influenza vaccination among patients with chronic obstructive pulmonary disease: a population-based cross-sectional study. Asia Pac J Public Health. 2017:29(7):560-8

16. Kwon DS, Kim K, Park SM. Factors associated with influenza vaccination coverage among the elderly in South Korea: the Fourth Korean National Health and Nutrition Examination Survey (KNHANES IV). BMJ Open. 2016;6(12):e012618

17. Oh MG, Han MA, Yun NR, Park J, Ryu SY, Kim DM, Choi SW. A PopulationBased, Nationwide Cross-Sectional Study on Influenza Vaccination Status among Cancer Survivors in Korea. Int J Environ Res Public Health. 2015;12(8):10133-49.

18. Paget J, Caini S, Cowling B, Esposito S, Falsey AR, Gentile A, Kyncl J, Maclntyre C, Pitman R, Lina B. The impact of influenza vaccination on the COVID-19 pandemic? Evidence and lessons for public health policies. Vaccine. 2020;38(42):6485-6.

19. Jehi L, Ji X, Milinovich A, Erzurum S, Rubin BP, Gordon S, Young JB, Kattan MW. Individualizing Risk Prediction for Positive Coronavirus Disease 2019 Testing: Results From 11,672 Patients (1931-3543 (Electronic)).

20. Li Q, Tang B, Bragazzi NL, Xiao Y, Wu J. Modeling the impact of mass influenza vaccination and public health interventions on COVID-19 epidemics with limited detection capability. Math Biosci. 2020:325:108378.

21. Ganganwar $V$. An overview of classification algorithms for imbalanced datasets. Int J Emerg Technol Adv Eng. 2012;2(4):42-7.

\section{Publisher's Note}

Springer Nature remains neutral with regard to jurisdictional claims in published maps and institutional affiliations.
Ready to submit your research? Choose BMC and benefit from

- fast, convenient online submission

- thorough peer review by experienced researchers in your field

- rapid publication on acceptance

- support for research data, including large and complex data types

- gold Open Access which fosters wider collaboration and increased citations

- maximum visibility for your research: over 100M website views per year

At BMC, research is always in progress.

Learn more biomedcentral.com/submissions 